REGHIN,M.Y.; OTTO, R.F.; OLINIK, J.R.; JACOBY, C.F.S.; OLIVEIRA, R.P. Vernalização em bulbos e efeito no rendimento e potencial fisiológico de sementes de cebola. Horticultura Brasileira, Brasília, v.23, n.2, p.294-298, abr-jun 2005.

\title{
Vernalização em bulbos e efeito no rendimento e potencial fisiológico de sementes de cebola
}

\author{
Marie Y. Reghin; Rosana F. Otto; Jean Ricardo Olinik; Carlos Felipe S. Jacoby; Rafael P. de Oliveira \\ UEPG. Depto. Fitotecnia e Fitossanidade, Av.Carlos Cavalcanti 4748, 84030-900 Ponta Grossa-PR; E-mail:freghin@convoy.com.br
}

\begin{abstract}
RESUMO
O estado do Paraná contribui significativamente para o abastecimento de cebola no Brasil. No entanto, a atividade de produção de sementes está muito aquém do desejado, o que torna urgente a necessidade de pesquisa nessa área. Este experimento foi conduzido em Ponta Grossa (PR) e teve como objetivo avaliar o efeito de períodos de vernalização em bulbos de tamanhos diferentes, no rendimento e potencial fisiológico de sementes de cebola da cultivar Crioula. O delineamento experimental utilizado foi de blocos casualizados, com quatro repetições; os tratamentos foram dispostos segundo esquema fatorial $4 \times 2$, sendo quatro períodos de vernalização a $5^{\circ} \mathrm{C}$ (controle, 25; 35 e 45 dias) e dois tamanhos de bulbos, T1 $(6,9 \mathrm{~cm}$ de diâmetro) e T2 $(4,8 \mathrm{~cm})$. A vernalização contribuiu para o aumento do rendimento de sementes. Quanto maior o período de tratamento, maior o percentual de umbelas com flores, o número de hastes florais, o número total de cápsulas e de cápsulas com sementes. Estes componentes promoveram maior rendimento de sementes por umbela, tanto em número como em peso, apresentando os períodos de 35 e 45 dias como os mais favoráveis. No entanto, no rendimento de sementes por planta, prevaleceu apenas a superioridade do período de 45 dias. O potencial fisiológico das sementes não foi afetado pelos tratamentos, tendo sido observados resultados acima de $80,0 \%$ no teste de primeira contagem e acima de $90,0 \%$ no percentual de germinação, independentemente do tratamento. $\mathrm{O}$ tamanho do bulbo maior (T1) apresentou efeito significativo apenas no número de hastes florais e de flores fecundadas, que contribuíram para o maior rendimento de sementes por planta, quando comparado ao tamanho $\mathrm{T} 2$.
\end{abstract}

Palavras-chave: Allium cepa L., frigorificação de bulbos, produção de sementes.

\begin{abstract}
Vernalization of bulbs and the effect on yield and physiological potential of onion seeds

The Paraná State (Brazil) significantly contributes to the supply of onions in Brazil. However, the seed yield activity is far below that desired, giving rise to a need for research in this area. This experiment was conducted in Ponta Grossa county to evaluate the effect of vernalization periods on different sized bulbs on seed yield and physiological potential of onion seeds, cv. Crioula. The experimental design was of randomized blocks with four replications; the treatments followed a factorial scheme $4 \times 2$, with four vernalization periods at $5^{\circ} \mathrm{C}$ (control, 25; 35 and 45 days) and two sizes of bulbs (T1 with $6,9 \mathrm{~cm}$ of diameter) and $\mathrm{T} 2(4,8 \mathrm{~cm})$. The vernalization contributed to an increase of seed yield. The longer the treatment period, the higher the percentage of umbels, number of seed stalks, total number of capsules and capsules with seeds. These components contributed to higher seed yield per umbel, both in number and weight, the periods of 35 and 45 days being the most favorable. However, on the seed yield per plant, the period of 45 days prevailed. The seed physiological potential was not affected by the treatment. Germination was higher than $80,0 \%$ on the first test counting and higher than $90,0 \%$ in the last count, independent of treatment. Bigger bulbs (T1) produced greater number of flower stalks and larger amounts of fertilized flowers. These two parameters contributed to a higher seed yield per plant when compared to T2.
\end{abstract}

Keywords: Allium cepa L., bulbs frigorification, seed yield.

(Recebido para publicação em 10 de maio de 2004 e aceito em 9 de março de 2005)

$\mathrm{N}$ o estado do Paraná, a produção de cebola está concentrada em mais de 1.000 hectares nos municípios de Imbituva e Irati, que respondem como áreas de relevância de acordo com a safra 2001/2002, com uma área cultivada de 5.990 hectares (SEAB/DERAL, 2002). Embora a produção de bulbos seja uma das principais atividades de subsistência, a produção de sementes não tem acompanhado essa evolução no Estado.

Um dos fatores principais para obtenção de alto rendimento de sementes é a ocorrência de máxima porcentagem de florescimento. A temperatura é o principal fator que condiciona $o$ florescimento da cebola, sendo a faixa de $9-13^{0} \mathrm{C}$ considerada favorável para indução floral (BREWSTER, 1977; 1982; CURRAH; PROCTOR, 1990).

A necessidade de frio para induzir o florescimento é a principal dificuldade na produção de sementes de cebola em muitos países tropicais (KIMANI et al., 1994). Conseqüentemente, a vernalização artificial tem sido utilizada para induzir o florescimento em cebola. No Brasil, apenas os estados de Santa Catarina e Rio Grande do Sul teriam condições de produção de sementes sem a vernalização artificial dos bulbos-mãe (MULLER; CASALI, 1982). Conforme Thomazelli et al. (1990), as condições térmicas do Planalto
Catarinense durante o inverno e primavera permitem que se processe a vernalização natural das plantas de cebola, com emissão de bom número de umbelas por planta.

A necessidade de vernalização já foi constatada no Nordeste (AGUIAR, 1984), Minas Gerais (SILVA et al., 1980) e em São Paulo (COSTA; DIAS, 1967), para a maioria das cultivares.

A vantagem da vernalização artificial é a rapidez e uniformidade de florescimento (COSTA; DIAS, 1967, KIMANI et al., 1997). Para Branca e Ruggeri (1994) existe interação da temperatura e do fotoperíodo para ocorrer florescimento. Os autores observaram 
que as cultivares apresentaram florescimento em condições acima de 1.900 horas de frio com temperaturas abaixo de $12^{\circ} \mathrm{C}$. No entanto, essa quantidade de frio foi insuficiente quando as plantas desenvolveram-se em condições de comprimento de dia de 10 horas, com poucas cultivares apresentando florescimento. Bulbos submetidos a um período de vernalização acima de 1.900 horas, com temperaturas abaixo de $12^{\circ} \mathrm{C}$, e plantas desenvolvendo em condições de comprimento de dia de 12,9 horas produziram maior quantidade de sementes.

A resposta ao florescimento deve depender de uma série de fatores, entre eles, da cultivar, do clima do local de produção, da temperatura para a indução floral, da época de plantio e tamanho dos bulbos-mãe (MELO; RIBEIRO, 1990). O tamanho do bulbo é outra característica importante diretamente relacionada ao potencial de rendimento de sementes por planta. Quanto maior for o bulbo, maior é a produção de sementes (MELLO et al.,1974; RUSEV, 1980; GARCIA et al.; 1982; TOMAR, 2001).

A ausência de dados sobre a produção de sementes de cebola utilizandose a vernalização de bulbos no estado do Paraná, motivou a realização do presente trabalho que buscou avaliar períodos de vernalização em bulbos de tamanhos diferentes no rendimento e no potencial fisiológico de sementes da cultivar Crioula.

\section{MATERIAL E MÉTODOS}

O experimento foi conduzido em Ponta Grossa (PR), localizado à altitude de $880 \mathrm{~m}$ e em solo do tipo CAMBISSOLO HÁPLICO Tb Distrófico, de textura argilosa. O delineamento experimental utilizado foi de blocos casualizados com quatro repetições com tratamentos em esquema fatorial $4 \times 2$, sendo quatro períodos de vernalização a $5^{\circ} \mathrm{C}$ (controle sem vernalização, 25; 35 e 45 dias de vernalização) e dois tamanhos de bulbos, T1 (6,9 cm de diâmetro) e T2 (4,8 $\mathrm{cm})$ da cultivar Crioula. A primeira amostra de bulbos foi colocada na câmara fria (com temperatura de $5^{\circ} \mathrm{C} \pm 1 \mathrm{e}$ $80,0-85,0 \%$ de umidade relativa) a partir de 31/03/2003 para o tratamento de vernalização de 45 dias e, a cada dez dias (10/04 e 20/04), repetiu-se o mesmo procedimento para os tratamentos de 35 e 25 dias de vernalização, respectivamente. Os bulbos do controle permaneceram em condições de temperatura ambiente (média de $\pm 22^{\circ} \mathrm{C}$ ) de laboratório. No campo, cada parcela apresentou três linhas, com doze plantas por linha e dispostos no espaçamento de 1,10x0,20 m, totalizando 36 plantas. O plantio foi realizado em 16/05/2003 em área que recebeu adubação, de acordo com a análise química do solo, com a fórmula química 5-20-10 na quantidade de $100 \mathrm{~g} / \mathrm{m}^{2}$. Procederam-se adubações nitrogenadas em cobertura com uréia $(20$ g/m) aos 80; 100 e 120 dias do plantio e a área foi mantida com irrigação por aspersão. As avaliações foram conduzidas em quatro plantas marcadas da linha central. O número de umbelas com flores abertas e o número de hastes florais foram avaliadas aos 153 dias.

A colheita foi feita em etapas, de acordo com a maturação das sementes. Cada umbela foi colhida adotando-se como ponto de colheita quando $10 \%$ de sementes estavam expostas nas umbelas (THOMAZELLI et al., 1990; REGHIN et al., 2004), no período de 219 a 241 dias do plantio. Em cada colheita, cortou-se a umbela com tesoura, depositando-a separadamente em sacos de papel e identificando-a se proveniente da planta $1 ; 2 ; 3$ ou 4 , respectivamente, até a colheita da última umbela de cada planta marcada. Após serem conduzidas para o laboratório, as umbelas foram colocadas sobre jornal em um balcão. Numa amostra de três umbelas por parcela, em cada uma procedeu-se a separação e contagem de flores fecundadas e não fecundadas. Nas flores fecundadas (cápsulas), as sementes foram retiradas e acondicionadas em gerbox, separadamente de 1 até 6 sementes. Dessa forma, as sementes foram separadas e classificadas com numeração de $1 ; 2 ; 3 ; 4$; 5 ou 6 . Foram avaliados o número total de sementes, número de sementes por cápsulas, massa de 100 sementes e massa de sementes por umbela. Para as demais umbelas da planta, fez-se a trilha com utilização de peneiras para separação de sementes e estas, adicionadas às sementes das três umbelas anteriores, constituíram o rendimento de sementes por planta. Para avaliar a qualidade fisiológica das sementes, foram realizados testes de germinação, segundo as orientações prescritas nas Regras de Análises de Sementes (BRASIL, 1992). Utilizaram-se quatro repetições de 100 sementes para cada tratamento. O vigor foi avaliado pela contagem das plântulas normais do teste de germinação, no sexto dia (NAKAGAWA, 1994) e a germinação no décimo segundo dia, sob temperatura de $20^{\circ} \mathrm{C}$.

Os dados foram submetidos à análise de variância e, em casos de significância, utilizou-se o teste de Tukey a 5\% de probabilidade, para comparar as médias dos tratamentos.

\section{RESULTADOS E DISCUSSÃO}

O ciclo reprodutivo da cebola durou 220 dias, do plantio à data da primeira colheita de sementes. Entretanto, esperava-se que a colheita pudesse ser realizada mais cedo, principalmente como conseqüência da vernalização. Constatou-se que o ciclo foi longo, se considerarmos que Thomazelli et al. (1990), Reghin et al. (2004), ao trabalharem com a mesma cultivar Crioula, sem vernalização de bulbos, obtiveram média de ciclo de 179 dias em Caçador (SC) e de 180 dias, em Imbituva (PR), respectivamente.

Observou-se que a fase do crescimento posterior ao plantio até o início do florescimento foi longa, de 122 dias. Desta forma, o florescimento praticamente ocorreu na mesma época do método sem vernalização, pois conforme Melo e Ribeiro (1990) o florescimento nesse método ocorre em meados de outubro, com plantio realizado em julho.

Um dos fatores que pode ter contribuído para esse alongamento do ciclo foi a época de plantio. A brotação pósplantio e a iniciação floral, embora tivessem ocorrido em temperatura favoravelmente baixa de maio, o fotoperíodo nesta época foi curto. Ademais, Aguiar et al. (1983) recomendam o plantio de bulbos vernalizados no vale do São Francisco em 10 e 11 de junho.

Os resultados de Branca e Ruggeri (1994) reforçam essa consideração, ao 
Tabela 1. Porcentagem de umbelas com flores aos 153 dias, número de hastes florais, total de flores, de flores fecundadas (cápsulas) e não fecundadas, número de sementes por cápsula, rendimento de sementes em número e peso por umbela e por planta, massa de 100 sementes, vigor (\% de germinação na $1^{\text {a }}$ contagem) e germinação de sementes de cebola, cultivar Crioula, em função dos períodos de vernalização. Ponta Grossa, UEPG, 2003.

\begin{tabular}{|c|c|c|c|c|c|c|c|c|c|c|c|c|}
\hline $\begin{array}{c}\text { Vernaliza- } \\
\text { ção } \\
\text { (dias) }\end{array}$ & $\begin{array}{c}\text { Umbelas } \\
\text { c/ flores } \\
(\%)\end{array}$ & $\begin{array}{l}\text { Hastes } \\
\text { florais } \\
\text { (n) }\end{array}$ & $\begin{array}{l}\text { Total de } \\
\text { flores }(n)\end{array}$ & $\begin{array}{l}\text { Flores } \\
\text { fecund. } \\
\text { (n) }\end{array}$ & $\begin{array}{l}\text { Flores } \\
n / \text { fec. (n) }\end{array}$ & $\begin{array}{l}\text { Sementes } \\
\text { Icápsula } \\
\text { (n) }\end{array}$ & $\begin{array}{c}\text { Sementes } \\
\text { /umbela } \\
\text { (n) }\end{array}$ & $\begin{array}{l}\text { Sementes } \\
\text { /umbela } \\
\text { (g) }\end{array}$ & $\begin{array}{l}\text { Sementes } \\
\text { /planta (g) }\end{array}$ & $\begin{array}{c}\text { M. de } \\
100 \text { stes } \\
(\mathrm{mg})\end{array}$ & $\begin{array}{l}\text { Vigor } \\
(\%)\end{array}$ & $\begin{array}{c}\text { Germinação } \\
(\%)\end{array}$ \\
\hline controle & $8,75^{*} \mathrm{c}$ & $5,44^{*} b$ & $529,12^{*} \mathrm{c}$ & $293,88^{*} b$ & $235,24^{*} b$ & $2,40^{*} a$ & $713,71^{*} b$ & 2,12 * b & $11,91^{*} \mathrm{c}$ & $300,95^{*} a$ & $82,0^{*} a$ & $93,0^{*} a$ \\
\hline 25 & $32,14 \mathrm{~b}$ & $6,59 a b$ & $582,66 \mathrm{c}$ & $372,71 \mathrm{~b}$ & 209,95 b & $2,55 \mathrm{a}$ & $960,67 \mathrm{~b}$ & $3,04 \mathrm{~b}$ & $22,53 \mathrm{bc}$ & $326,58 \mathrm{a}$ & 83,6 a & 94,8 a \\
\hline 35 & $42,47 a b$ & $7,22 \mathrm{a}$ & $754,41 \mathrm{~b}$ & $518,91 \mathrm{a}$ & $235,50 \mathrm{~b}$ & $2,69 a$ & $1.385,62$ a & 4,71 a & $31,75 a b$ & 351,26 a & 86,0 a & 95,8 a \\
\hline 45 & $51,49 a$ & $7,62 \mathrm{a}$ & $910,75 a$ & 552,63 a & $358,12 a$ & $2,83 a$ & $1.603,33 \mathrm{a}$ & 5,23 a & $40,53 a$ & $330,34 \mathrm{a}$ & 89,0 a & $96,2 \mathrm{a}$ \\
\hline$\overline{\text { C.V. (\%) }}$ & 28,15 & 18,99 & 13,19 & 13,99 & 26,11 & 12,74 & 19,38 & 21,11 & 27,98 & 12,75 & 9,82 & 3,54 \\
\hline
\end{tabular}

*Médias seguidas da mesma letra na coluna não diferem significativamente entre si no nível de $5 \%$ de probabilidade pelo teste de Tukey.

observarem na Itália, cultivares com comportamento mais favorável quando submetidas a mais que 1.900 horas abaixo de $12^{\circ} \mathrm{C}$ e comprimento de dia de 12,9 horas. Por outro lado, com a mesma quantidade de frio e comprimento de dia de 10,0 horas, somente algumas cultivares floresceram. Para a cultivar Crioula, além da necessidade de temperatura baixa, é possível que o fotoperíodo adequado ou ideal seja mais longo que o do experimento para estimular um rápido florescimento. Outro fator que pode ter contribuído para a demora no florescimento foi a exposição em temperatura de vernalização a $5^{\circ} \mathrm{C}$, pois Kimani et al. (1994) observaram que bulbos vernalizados a $10^{\circ} \mathrm{C}$ resultaram em florescimento mais precoce, com maior número de umbelas e produção de sementes do que os bulbos tratados em temperatura mais baixa $\left(5^{\circ} \mathrm{C}\right)$ ou mais alta $\left(13,4\right.$ e $\left.21,9^{\circ} \mathrm{C}\right)$.

Os resultados demonstram que cada cultivar tem a sua exigência de horas de frio para o florescimento, assim como a temperatura ótima de vernalização, mas o frio isoladamente, não é suficiente para assegurar totalmente o subseqüente desenvolvimento do processo reprodutivo.

Mesmo em se tratando de bulbos vernalizados artificialmente, o plantio antecipado no mês de maio não promoveu vantagem adicional na característica de precocidade de florescimento para a cultivar Crioula.

Embora com longo período de desenvolvimento até o início do florescimento (122 dias), a vernalização foi efetiva no aumento do percentual de florescimento, comparado ao controle. Aos 153 dias do plantio, quanto maior o período de vernalização maior foi o percentual de umbelas florescidas (Tabela 1). Com 45 dias de vernalização, obteve-se $51,49 \%$ de florescimento, enquanto no controle, apenas $8,75 \%$. No controle, as umbelas apresentaram pleno florescimento somente no final de outubro (168 dias). O período de florescimento foi longo, em torno de dois meses.

O número de hastes florais por planta variou em função dos períodos de vernalização e do tamanho do bulbo. Os maiores períodos (35 e 45 dias) estimularam a emissão de maior número de hastes florais por planta, o que é uma resposta vantajosa no rendimento de sementes, diferindo consideravelmente do controle (Tabela 1) e concordando com os resultados obtidos por Aguiar et al. (1983) e Aguiar (1984). Considerando a média obtida por Thomazelli et al. (1990), de 4,91 hastes florais no processo sem vernalização, após várias safras com a mesma cultivar Crioula, e o valor de 5,44 obtido no controle deste experimento, podemos considerar que esta técnica é eficiente, aumentando o número de hastes para 7,22 com 35 dias de vernalização e para 7,62 com 45 dias.

$\mathrm{O}$ uso de bulbos de maior tamanho (T1), por ser mais rico em reserva, promoveu um aumento significativo no número de hastes florais (Tabela 4), coincidindo com resultados de Garcia et al. (1982). Quando for possível, é preferível optar por bulbos maiores, mas o custo financeiro poderá ser fator limitante, principalmente neste método em que há necessidade de armazenar os bulbos em câmara fria para a vernalização. Tomar (2001) recomenda como os mais favoráveis para produção de sementes, bulbos com peso entre 50 $60 \mathrm{~g}$ e 4,5-5,0 cm de diâmetro.

Entre as características avaliadas após a colheita, observou-se que o número total de flores na umbela foi incrementado em função do aumento do período de vernalização. O período de 45 dias de vernalização demonstrou o melhor resultado. E o de 35 dias apresentou comportamento intermediário. $\mathrm{O}$ período de 25 dias e o controle apresentaram os menores valores. Com 45 dias de vernalização, houve aumento expressivo de 381,6 flores quando comparada com o controle. $\mathrm{O}$ fato importante é que os resultados observados nos tratamentos de vernalização demonstram que é possível um aumento no total de flores fecundadas (Tabela 1).

Segundo Melo e Ribeiro (1990), a umbela pode ter de 50 a 2000 flores. Após a fecundação, há o desenvolvimento da semente e, desde que a polinização seja eficiente, poderá haver a formação máxima de seis sementes por flor, o que chamamos de cápsula. Portanto, com a possibilidade de totalizar 2000 flores por umbela e tendo obtido a média de 694,2 , o percentual de $34,7 \%$ é baixo, considerando a potencialidade de produção da umbela (Tabela 1). O percentual de $37,8 \%$ de flores não fecundadas em relação ao total de flores produzidas (Tabela 1), permite constatar que a ausência de polinização pode ser um dos componentes de rendimento que pode ser melhorado com o aumento de agentes polinizadores na área de produção.

Embora o tratamento com 45 dias de vernalização tenha apresentado o maior valor no número de flores fecundadas, este mesmo tratamento apresentou o 
maior valor em flores não fecundadas. Levando em consideração o tratamento em que as parcelas floresceram primeiro e a quantidade das umbelas florescidas serem menores proporcionalmente aos demais tratamentos, é possível inferir que as umbelas não foram atrativas o suficiente para garantir eficiência na polinização. Witter e Blochtein (2003) observaram que somente $48 \%$ das flores de Crioula Alto Vale desenvolveram cápsulas e apontaram como causa da perda a falta de polinização. Outra característica que reforça a necessidade de melhoria da polinização é o número de sementes por cápsula. Ainda que diferenças significativas não tenham sido apresentadas, a média de 2,6 sementes por cápsula é baixa, considerando o potencial de produção de até seis sementes (Tabela 1). As maiores contribuiçõos no rendimento de sementes foram em ordem decrescente, de cápsulas com $3 ; 4 ; 2 ; 5 ; 1$ e 6 sementes, respectivamente, sendo as três primeiras as mais significativas, contribuindo com $77 \%$ do total produzido. Por outro lado, as cápsulas com 5 e 6 sementes representaram apenas $11,4 \%$ e $3,8 \%$, respectivamente (Figura 1). Sampaio et al. (1998) também observaram no Rio Grande do Sul que cápsulas com 3 sementes ocorreram com maior freqüência (28\%).

A maior quantidade de hastes florais e de flores fecundadas nos tratamentos com 35 e 45 dias de vernalização asseguraram os maiores rendimentos de semente por umbela, tanto em número como em peso, quando comparados com 25 dias e controle (Tabela 2).

O rendimento de $4,7 \mathrm{~g}$ por umbela (35 dias) e de 5,2 g (45 dias) nesses dois tratamentos demonstra a importância da vernalização na produção de sementes de cebola, pois a produtividade, tanto em número como em peso de sementes foi bastante expressiva, quando comparada ao controle, com 2,1 g por umbela (Tabela 1). O rendimento do controle foi bastante semelhante à média de $2,9 \mathrm{~g}$ obtida em Santa Catarina, após várias safras com a cultivar Crioula, sem vernalização dos bulbos (THOMAZELLI et al., 1990).

Os períodos de 35 e 45 dias de vernalização foram eficientes relativa-

Tabela 2 Porcentagem de umbelas com flores, número de hastes florais, total de flores, de flores fecundadas e não fecundadas, sementes por cápsula e por umbela, massa de sementes por umbela, por planta e de 100 sementes, vigor e porcentagem de germinação, em função do tamanho de bulbos ( $\mathrm{T} 1=6,9 \mathrm{~cm}$ e T2=4,8 cm), na produção de sementes de cebola, cultivar Crioula. Ponta Grossa, UEPG, 2003.

\begin{tabular}{lrr}
\hline \multicolumn{1}{c}{ Características } & Tamanho 1 & Tamanho 2 \\
\hline Porcentagem de umbelas com flores & $34,26 \mathrm{~A}^{*}$ & $33,17 \mathrm{~A}^{*}$ \\
N. de hastes florais/planta & $7,50 \mathrm{~A}$ & $5,94 \mathrm{~B}$ \\
N. total de flores & $723,29 \mathrm{~A}$ & $680,75 \mathrm{~A}$ \\
N. de flores fecundadas & $461,96 \mathrm{~A}$ & $407,10 \mathrm{~B}$ \\
N. de flores não fecundadas & $261,33 \mathrm{~A}$ & $258,08 \mathrm{~A}$ \\
N. de sementes por cápsula & $2,64 \mathrm{~A}$ & $2,60 \mathrm{~A}$ \\
N. de sementes por umbela & $1233,75 \mathrm{~A}$ & $1097,94 \mathrm{~A}$ \\
Massa de sementes por umbela (g) & $4,01 \mathrm{~A}$ & $3,55 \mathrm{~A}$ \\
Massa de sementes por planta (g) & $31,42 \mathrm{~A}$ & $21,94 \mathrm{~B}$ \\
Massa de 100 sementes (mg) & $332,93 \mathrm{~A}$ & $321,63 \mathrm{~A}$ \\
Vigor (\% de germinação na1a contagem) & $85,9 \mathrm{~A}$ & $84,4 \mathrm{~A}$ \\
Porcentagem de germinação & $95,1 \mathrm{~A}$ & $94,8 \mathrm{~A}$ \\
\hline
\end{tabular}

*Médias seguidas da mesma letra na linha não diferem significativamente entre si no nível de $5 \%$ de probabilidade pelo teste de Tukey.

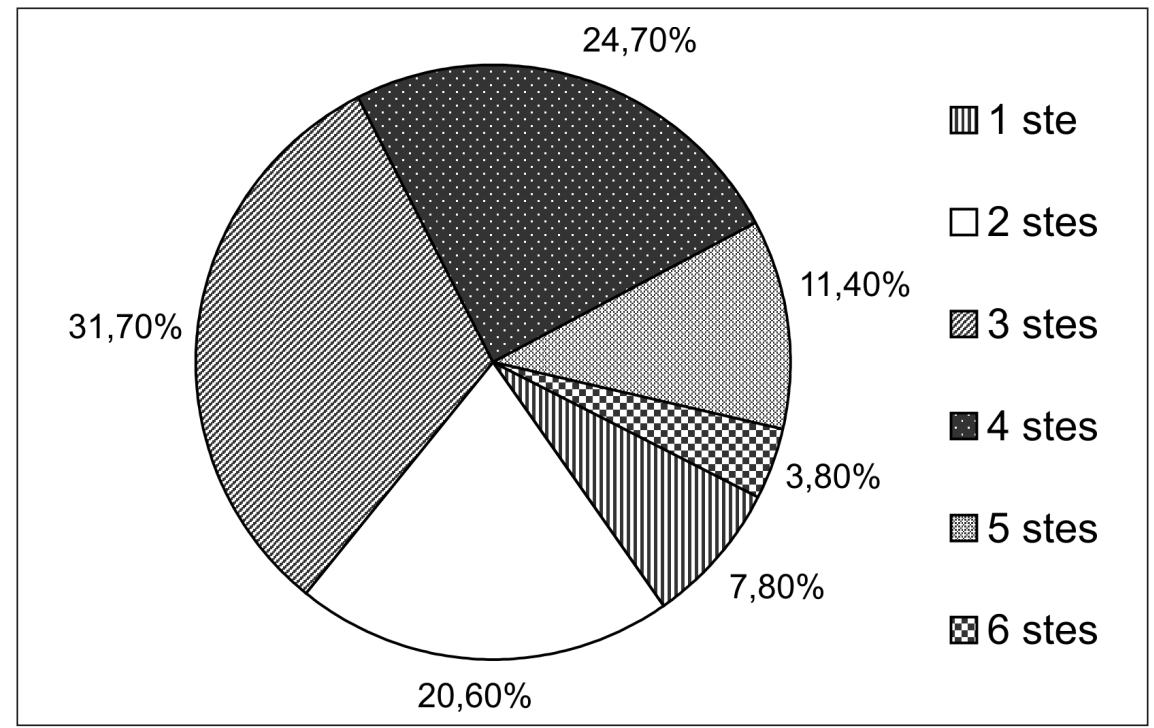

Figura 1. Contribuição no rendimento de sementes de cebola (\%), de cápsulas com $1 ; 2 ; 3$; 4; 5 e 6 sementes, da cultivar Crioula. Ponta Grossa, UEPG, 2003.

mente ao aumento do número de hastes florais, a taxa de florescimento das umbelas e consequentemente, o rendimento de sementes por umbela. Por sua vez, o rendimento de sementes por planta demonstrou que esse comportamento superior prevaleceu para os tratamentos de vernalização por 45 e 35 dias diferindo do de 25 dias e do controle, apesar do tratamento por 35 dias não diferir do resultado obtido com 25 dias de vernalização (Tabela 1).

Embora tenha ocorrido uma tendência de aumento da massa de 100 semen- tes de acordo com o aumento do período de vernalização, a diferença observada não foi significativa (Tabela 1). Aguiar et al. (1983) relataram diminuição gradativa da massa média de acordo com a diminuição do período de vernalização, fato este observado, de um modo geral, nas cultivares estudadas.

Igualmente, os tratamentos utilizados não afetaram o potencial fisiológico das sementes, ou seja, os percentuais observados de vigor e de germinação demonstraram que independentemente da vernalização ou do tamanho do bul- 
bo plantado, as sementes obtidas apresentaram alta qualidade. Os resultados do teste de primeira contagem da germinação (vigor), apontaram valores acima de $80,0 \%$ e a porcentagem de germinação acima de $90 \%$, em qualquer tratamento (Tabela 1).

O tamanho maior do bulbo (T1) apresentou tendência positiva em todas as características de rendimento de sementes quando comparada ao tamanho T2 (Tabela 2). No entanto, uma diferença estatisticamente significativa foi observada apenas nas características de número de umbelas/planta e no número de flores fecundadas, as quais contribuíram para o maior rendimento de sementes por planta quando bulbos de tamanho T1 (6,9 cm de diâmetro) foram usados.

\section{LITERATURA CITADA}

AGUIAR, P.A.A.; D'OLIVEIRA, L.O.B.; ASSUNÇÃO, M.V. Vernalização de bulbos na produção de sementes de cebola na região do submédio São Francisco. Pesquisa Agropecuária Brasileira, Brasília, v.18, n.7, p.741-746, 1983.

AGUIAR, P.A.A. Período de vernalização dos bulbos de cebola para produção de sementes, no Nordeste do Brasil. Pesquisa Agropecuária Brasileira, Brasília, v.19, n.2, p.197-200, 1984.

BRANCA, F.; RUGGERI, A. Reproductive response of onion seed plants to photothermal conditions. Acta Horticulturae, v.362, p.25-33, 1994.
BRASIL, Ministério da Agricultura e Reforma Agrária. Regras de Análise de Sementes, Brasília: MAARA/SNDA/DNDV/CLAV, 365 p. 1992.

BREWSTER, J.L. The physiology of the onion. HorticulturalAbstracts, v. 47, n. 2, p. 103-112, 1977. BREWSTER, J.L. Growth, dry matter partition and radiation interception in an overwintered bulb onion crop. Annual Botany, v.49, p.609-617, 1982. COSTA, C.P.; DIAS, M.S. Comparação do método de frigorificação vs. florescimento em condições naturais e suas consequências para o melhoramento da cebola nas condições de estado de São Paulo. Relatório de Ciências do Instituto de Genética, ESALQ, USP, n.1, p.94-97, 1967.

CURRAH, L.; PROCTOR, F.J. Onions in the tropical regions. Bulletin n.35. Natural Resources Institute, ODA, U.K. 1990.

GARCIA, A.; PATELLA, A.E.; FELICIANO, A. Efeito da época de plantio, tamanho de bulbo e espaçamento em cebola para sementes. Pelotas, EMBRAPA-UEPAE Cascata, 1982, 43 p. (EMBRAPA-UEPAE Cascata, Boletim Pesquisa, 1). KIMANI, P.M.; KARINKI, J.L.W.; PETERS,R.; RABINOWITCH, H.D. Potential of onion seed production in a tropical environment. Acta Horticulturae, v.358, p.341-349, 1994.

MELLO, V.D.C.; ROCHA, F.F.; FILES, P. Produção de sementes de cebola (Allium cepa L.) através de bulbinhos. Revista de Olericultura, Santa Maria, n.14, p.195-196, 1974.

MELO, P.C.; RIBEIRO, A. Produção de sementes de cebola: cultivares de polinização aberta e híbridos. In: CASTELLANE, P.D.; NICOLOSI, W.M.; HASEGAWA, M. (coord.). Produção de sementes de hortaliças. Jaboticabal, FCAV/ FUNEP, 1990. p.15-59.

MULLER, J.J.V.; CASALI, V.W.D. Produção de sementes de cebola (Allium cepa L.). Florianópolis, EMPASC, 1982. 64 p. (EMPASC. Boletim Técnico, 16)
NAKAGAWA, J. Testes de vigor baseados na avaliação das plântulas. In: VIEIRA, R.D.; CARVALHO, N.M. Testes de vigor em sementes. Jaboticabal: FUNEP, 1994. 164 p.

REGHIN, M.Y.; DALLA PRIA, M.D.; OTTO, R.F.; VINNE, J. Épocas de colheita de umbelas e comprimento da haste floral no rendimento e no potencial fisiológico de sementes de cebola. Horticultura Brasileira, Brasília, v.22, n.02, p.286289, 2004.

RUSEV, D. Effect of bulb size and planting date on seed yield and quality in the onion cultivar Lyaskovski 58. Horticultural Abstracts, v.50, n.7, p.431, 1980.

SAMPAIO, T.G.; SAMPAIO, N.V.; SOARES, P.F.

Estudo de componentes do rendimento na produção de sementes de cebola (Allium cepa L.). Ciência Rural, Santa Maria, v.3, n.1, p.1-7, 1998.

SEAB/DERAL. Valor Bruto da Agropecuária Paranaense. Disponível: site do Governo do Estado do Paraná, 2002. <http:/www.pr.govbr/seab/ deral.> Acesso em 03/01/2004.

SILVA, R.F.; CASALI, V.W.D.; VIGGIANO, J. Produção de sementes de cebola. Informe Agropecuário, Belo Horizonte, v.6, n.62, p.32-35, 1980.

THOMAZELLI, L.F.; BIASI, J.;YOKOYAMA, S.; BECKER, W.; FAORO, I.D.; SILVA, A.C.F.; MULLER, J.J.V.; GUIMARÃES, D.R.;ZANINI NETO, J.A.; VIZZOTTO, V.J. Produção de sementes de cultivares selecionadas de cebola em Santa Catarina. Pesquisa agropecuária brasileira, Brasília, v.25, n.11, p.1607-1612, 1990.

TOMAR, B.S. Onion seed production. Indian Horticulture, v.46, n.2, p.14-16, 2001.

WITTER,S.; BLOCHTEIN,B. Efeito da polinização por abelhas e outros insetos na produção de sementes de cebola. Pesquisa agropecuária brasileira, Brasília, v.38, n.12, p.1399-1407, 2003. 